\title{
Intervención del propietario en la mejora cualitativa de la vivienda de interés social adjudicada por el gobierno: caso de la Ciudad Jardín, Tunja (Colombia)
}

\author{
Juan M. Mendoza-Vargas ${ }^{1}$, Víctor M. Burbano-Pantoja ${ }^{2 \star}$, y Hugo H. Mendoza-Vargas ${ }^{1}$ \\ (1) Univ. Pedagógica y Tecnológica de Colombia, Uptc. Av Central del Norte, Tunja Colombia. \\ (correo-e: juanmaria.mendoza@uptc.edu.co; 2hsmendoza@gmail.com) \\ (2) Facultad de Ciencias, Escuela de matemáticas, Univ. Pedagógica y Tecnológica de Colombia, Uptc. Av Central del \\ Norte, Tunja Colombia. (correo-e: victor.burbano@uptc.edu.co)
}

* Autor a quien debe ser dirigida la correspondencia.

Recibido Jun. 10, 2021; Aceptado Ago. 10, 2021; Versión final Sep. 1, 2021, Publicado Dic. 2021

\begin{abstract}
Resumen
En esta investigación se examina una urbanización de bajos ingresos para analizar viviendas con variaciones en el diseño inicial hechas por los propietarios. La Ciudad Jardín es una urbanización de vivienda de interés social (VIS) ubicada en una zona periférica de la ciudad de Tunja (Colombia) que fue adjudicada por el gobierno. La metodología se centró en un enfoque empírico-analítico cuantitativo de tipo observacionaldescriptivo. Los datos se recolectaron por medio de una encuesta a 78 propietarios de la urbanización. Los resultados muestran que el $29.49 \%$ de los propietarios indica que ha realizado mejoras en el baño y la cocina, el $7.69 \%$ en la construcción de paredes, el $28.21 \%$ en cambio de pisos, el $25.64 \%$ en instalaciones eléctricas y de agua, el $8.97 \%$ en servicios de internet. Se concluye que el esfuerzo económico realizado por los propietarios ha mejorado la habitabilidad y funcionabilidad de sus viviendas.
\end{abstract}

Palabras clave: adecuación; vivienda; periferia; planificación urbana; habitabilidad

\section{Owner renovations to qualitatively improve their low-income houses provided by the government: the case of La Ciudad Jardín, Tunja (Colombia)}

\begin{abstract}
In this study, a low-income housing urbanization is examined to analyze owner-made renovations to initial home designs. La Ciudad Jardín is a social interest housing $(\mathrm{SIH})$ urbanization provided by the government that is located in a peripheral area of the city of Tunja (Colombia). The methodology applied focused on a quantitative empirical-analytical approach that was observational and descriptive. Data were collected by surveying 78 urbanization home-owners. The results showed that home-owners made home improvements: $29.49 \%$ revamped bathrooms and kitchens, $7.69 \%$ constructed walls, $28.21 \%$ renovated floors, $25.64 \%$ repaired electrical installations and water pipes, and $8.97 \%$ invested on internet services. It is concluded that the economic efforts made by owners improved their home's habitability and functionality.
\end{abstract}

Keywords: renovation; housing; periphery; urban planning; habitability 


\section{INTRODUCCIÓN}

Esta investigación se enfocó al análisis de un modelo urbanístico de VIS, en el que se aprecia variaciones en el diseño inicial, tales variaciones fueron hechas por los usuarios como respuesta a una inadecuada vivienda que les fue asignada por el gobierno en la urbanización Ciudad Jardín de la ciudad de Tunja en Colombia; tal diseño contrastaba con los procesos de una buena planeación del crecimiento urbano y debían adecuarse a las características espaciales que han de corresponder a los programas de vivienda que pudieran satisfacer las necesidades de cada familia (Lehmann et al., 2015). En este contexto, cada usuario ha remodelado su vivienda sin desligarse del ordenamiento territorial reglamentado por la ley 388 de 1987, el cual incorpora las reformas al uso del suelo y define las características tanto internas como externas, incluidas las variaciones en las áreas construidas. Además, esta ley concede la participación al sector privado en la ejecución de los proyectos y deja en manos del Estado colombiano la financiación de los programas VIS en concordancia con las preferencias y capacidad económica del usuario, pero guardando el equilibrio en el crecimiento urbanístico de cada ciudad.

En este sentido, Muñoz et al. (2019) reconocen la autonomía que tienen los usuarios para mejorar progresivamente la funcionalidad y estética de su vivienda adjudicada por el gobierno, en concordancia con el contexto del barrio; ésta es una práctica recurrente a través de la historia y generalizada para este tipo de vivienda. Por otra parte, Valdivia (2018) agrega que, los desaciertos en la planificación de los programas VIS consisten en desconocer la naturaleza del contexto urbano y familiar, dejando en el olvido la inclusión de las condiciones humanas y psicológicas de quienes van a poblar el lugar. Por lo tanto, con este trabajo se pretende dar respuesta a la siguiente pregunta de investigación ¿Cómo es posible evidenciar las mejoras realizadas tanto internas como externas a la VIS por parte de los usuarios que habitan la urbanización ciudad Jardín del municipio de Tunja en Colombia? El análisis aporta los resultados de los cambios constructivos ejecutados por los usuarios que generaban conflictos tanto en la parte habitacional como en su entorno inmediato; por tanto las modificaciones realizadas se ajustaron a modelos de vivienda sostenibles y coherentes con la inversión.

En cuanto a la vivienda, Lehmann et al. (2015) afirman que en general, la planeación urbana separa el diseño de la construcción de la vivienda del ordenamiento urbano y no establece una ruta que formalice la evolución de las ciudades afectando a futuro a las administraciones de turno y a la población civil tanto en lo económico como en lo social; por lo tanto es perentorio que en el corto plazo se tenga en cuenta las necesidades espaciales del usuario en las fases de planificación y evaluación de los programas VIS. En particular, el acelerado crecimiento de la ciudad de Tunja también demanda construir centros de servicios accesibles para sus habitantes; en este sentido, la Ley 388 de1997 ordena a la administración territorial, diseñar con la comunidad la construcción de espacios que satisfagan las necesidades del sector incluyendo centros comerciales y demás servicios que causen impacto positivo en la comunidad, con sostenibilidad ambiental acorde a las costumbres y aspectos socioculturales. Asimismo, Murray y Clapham (2015) recomiendan a las administraciones locales incrementar la asignación de recursos económicos para cubrir las expectativas de vivienda de quienes aspiran a beneficiarse de la política pública de vivienda.

Por su parte, Chiodelli y Moroni (2017) consideran que en situaciones de complejidad política, las organizaciones del estado pueden optar por satisfacer necesidades de una población diversa, la cual ha de incluir víctimas de los conflictos y distintos credos religiosos, así como a beneficiarios con bajo nivel de ingreso pero con capacidad de articulación a tejidos sociales complejos. En este contexto, se hace necesario mejorar las condiciones actuales en las viviendas al crear un catálogo que mitigue el impacto negativo generado por la construcción en lotes reducidos, minimice los costos de las ampliaciones a que haya lugar para no desbordar la capacidad económica del usuario ni alterar significativamente el medio ambiente de la ciudad (García et al., 2018). En estas circunstancias, conviene evitar los asentamientos desorganizados que deterioran el urbanismo de la ciudad, con posibilidades inciertas de mejora ante la incapacidad de sus moradores de asumir los costos de variación en el diseño original y se ha de propender por el mejoramiento cualitativo de la vivienda basada en principios como la producción social del hábitat, la autoconstrucción y los procesos autogestionarios (Lehner y Gerscovich, 2021).

En cuanto a la calidad del hábitat, en América Latina y en Colombia, las políticas sociales de la VIS han pasado de una pretendida atención por parte del Estado, a un dejar de hacer o dejar pasar ante las dinámicas tanto del sector privado como de los sectores sociales; hoy prevalece el sentido mercantil y los intereses de los grupos que intervienen en la construcción de la vivienda; es decir, la política de VIS beneficia más a los propietarios de la tierra, los constructores y el sector inmobiliario que a los propios usuarios. En este escenario, la calidad de la VIS se ha visto cuestionada tanto en los reducidos espacios físicos construidos como en el componente social referido al grado de satisfacción del usuario por el espacio que habita (Mendoza et al., 2021); por lo tanto, se generan levantamientos periféricos y turbulentos en la comunidad, que reclama al gobierno la inclusión social y el reconocimiento del derecho a solucionar por sí misma el problema habitacional 
mediante la autogestión, cuando el principio de progresividad y las condiciones económicas del ente territorial no se lo permiten (Davies y Blanco, 2017).

Además, Timo et al. (2019) afirman que, el habitual paradigma de planificación urbana no ha sido suficiente para el cumplimiento en la ejecución de los programas VIS considerados internacionalmente como un derecho humano; por esto, en los procesos de gestión de las políticas sociales se hace necesario aplicar esquemas más eficientes que involucren la acción efectiva de los representantes de la administración pública, del sector constructor privado y de los potenciales beneficiarios. Estos aspectos han hecho que, los mismos usuarios hayan tenido que adelantar procesos de autoconstrucción al interior de sus VIS e instar al gobierno local para mejorar la infraestructura asociada con el entorno del barrio, la movilidad urbana, las vías de comunicación con el resto de la ciudad y la convivencia ciudadana con el propósito de mejorar la calidad de la VIS adjudicada y procurar un desarrollo urbano sostenible (Mendoza et al., 2020).

\section{OTROS ANTECEDENTES}

Estudios psicológicos comparativos sobre VIS con espacios físicos reducidos revelan que los usuarios presentan afectaciones negativas tanto en su salud como en sus aspectos socio-económicos y los beneficiarios de la urbanización Ciudad Jardín no han sido la excepción; impactados por la vulnerabilidad, la pobreza o la incapacidad de asumir el cumplimiento de sus obligaciones hipotecarias presionadas por las entidades de crédito o por los prestamistas particulares (Ruá et al., 2020), han decidido modificar mediante autoconstrucción el diseño inicial de la vivienda que les fue adjudicada. Algunos beneficiarios se han visto en la necesidad de ampliar la construcción en todo el lote de 42 metros cuadrados, otros construyeron pisos hacia arriba a fin de albergar a toda su familia, generar ingresos por arrendamiento o hacer emprendimientos como tiendas de barrio y alquiler de garaje, entre otros. Estas decisiones obedecen a que los gobiernos de turno no han sopesado el alcance de entregar VIS poco espaciosas a familias con baja capacidad financiera que ha vivido en condiciones extremas de vulnerabilidad.

También Valdivia (2018) agrega que, la planificación de la VIS ha desconocido la naturaleza del contexto de la planificación urbana para familias vulnerables y se ha enfocado más en la parte espacial que en la inclusión de las condiciones humanas y psicológicas; estos atributos inseparables son los que caracterizan la esencia personal de quienes habitaran el lugar e indican si se ha generado una mejora cualitativa de la vivienda. En varios casos, los restringidos recursos económicos de los habitantes, no les favorece acceder a los subsidios del estado ni a los créditos bancarios por su baja capacidad de ahorro, aspecto que los limita para el acceso financiero o el crédito en los bancos. En este sentido, Rolnik (2013) señala que los bajos ahorros no favorecen el desarrollo de la población cuando ésta envejece, pues es sabido que el ahorro es el apoyo más adecuado en las sociedades para alcanzar un nivel de estabilidad económico confiable a futuro; en Colombia el ahorro es menor al $20 \%$ de sus ingresos, considerado bajo en comparación con países de alto crecimiento, donde el ahorro supera el 35\% como lo ha indicado el Fondo Monetario Internacional en el año 2015.

La falta de cultura financiera manifestada en las bajas tasas de ahorro, no es tan relevante cuando se vive en situaciones de pobreza, puesto que el ahorro solo es viable cuando ya se ha dado solución a las necesidades básicas del grupo familiar. En este sentido, las políticas del microcrédito organizadas por el gobierno han sido insuficientes para los usuarios de la urbanización Ciudad Jardín, quienes han soportado el inconveniente de no poder incrementar sus ingresos mensuales, requisito exigido por el sector financiero para acceder a los créditos individuales de vivienda (Lehner y Gerscovich, 2021). Por esto, es prioritario que también el gobierno colombiano y el ente territorial brinde oportunidades para que los usuarios satisfagan sus necesidades básicas y de mejora de su vivienda a través de mecanismos de inserción económica de la población. En este contexto, las comunidades pueden mejorar su nivel de resiliencia con la ayuda gubernamental para acceder y disfrutar de los programas de vivienda a pesar de estar sumergidos en situaciones de pobreza (Vázquez et al., 2017).

Respecto al contexto legal de la planificación de los programas VIS, los gobiernos locales deben asumir compromisos de mejoramiento de los asentamientos humanos informales, legalmente autorizados pero que se encuentren en condiciones de deterioro; paralelamente la planificación debe reorientar el crecimiento urbano con procesos de mitigación de los impactos negativos en el medio ambiente (Soares et al., 2016). En este sentido, es necesaria la intervención del Estado colombiano, en la gestión integral de la política de la VIS, actuando como intermediario en el mercado del suelo y asumiendo las actividades de suministro de materiales para la autoconstrucción, neutralizando la responsabilidad tradicional de los particulares, quienes especulan e incumplen las estipulaciones de la contratación, observándose con frecuencia, el ofrecimiento de soluciones habitacionales de baja calidad. Asimismo, el gobierno ha de procurar un subsidio para que las familias de bajos ingresos puedan cubrir sus obligaciones financieras cuando carezcan de empleo y ha de servir de respaldo económico ante la dirección de las corporaciones financieras y los grupos económicos que han desarrollado la construcción del programa VIS adjudicado (Watt y Minton, 2016). 
Po otra parte, Carreño y Alfonso (2018) no justifican el acostumbrado sobreprecio del suelo conscientes de que el incremento no lo reflejan las operaciones matemáticas, en cambio, tal sobreprecio solo promueve la construcción de VIS en lotes con menos metros cuadrados; en este caso, el valor del suelo se incrementa fuertemente a expensas del poco control ejercido por la administración local sobre los propietarios de los predios; naturalmente estos incrementos se apartan de la realidad inflacionaria que afectan a otros mercados (Rolnik, 2013); también existe un aumento bien marcado en los costos de la construcción en Colombia y América Latina indicando que, dichos costos de edificación y de suelo construido suben a un promedio más elevado que el nivel de los salarios; lo que indica que la mayoría de las políticas y normas existentes respaldan más un ordenamiento de ciudad que al mismo financiamiento de las viviendas. Esta situación ha hecho que muchos usuarios hayan decido modificar o ampliar sus viviendas por sus propios medios acudiendo a la estrategia de autoconstrucción.

Este trabajo busca llamar la atención de la administración municipal sobre el rol jugado por los tradicionales gestores de la VIS, representados en desaciertos en la distribución de las VIS sobre espacios reducidos y la poca sostenibilidad del crecimiento urbano apreciado en la Ciudad Jardín a fin de que se supere el incumplimiento de las políticas de apoyo a la población más vulnerable (Libertun, 2018). Si bien es cierto que, los programas de vivienda gozan de una amplia trayectoria, las políticas aplicadas en Ciudad Jardín, han sido tergiversadas, los precios de la VIS son injustificados cuando ésta se ubica en la periferia con servicios públicos deficientes y en lotes de 42 metros cuadrados con un área construida de 10.5 metros cuadrados aproximadamente. El municipio de Tunja puede contribuir de mejor manera con la gente, sin apartarse del modelo de mercado ni de la normatividad política. En este sentido, con una oportuna intervención del estado en la planificación del crecimiento urbano, se puede hacer efectiva una mejora cualitativa de la VIS otorgada a los beneficiarios, sin que ellos tengan que hacer erogaciones económicas para mejorar la vivienda que ya se les ha adjudicado.

Como consecuencia de esto, la vivienda debe ser el inicio de la plena realización personal y familiar; al mismo tiempo, se puede constituir en la naturaleza que configura el contexto fundamental de la planificación urbana, teniendo en cuenta los aspectos socioeconómicos y las políticas promotoras del programa VIS; en este sentido, la entrega de una VIS con más metros construidos que guarde relación con el números de personas que conforman el núcleo familiar y con buenos servicios básicos debe ser una preocupación de los modelos de vivienda tradicionalmente estandarizados. Por consiguiente, la vivienda debe acomodarse al contexto natural y social de los potenciales usuarios; asimismo, ha de ofrecer un ambiente cómodo que dignifique a los beneficiarios con espacios interiores amplios, con atributos centrados en una buena accesibilidad, habitabilidad, comodidad, sostenibilidad y una adecuada comunicación con sus vecinos, de modo que, fomente los lazos de unión entre los habitantes del barrio, fortalezca las costumbres y tradiciones del sector (Adewale et al., 2020).

Visto desde la perspectiva urbana, la ciudad de Tunja guiada por el trazado colonial ha continuado su crecimiento hacia las periferias, marcando claros escenarios de segmentación socioeconómica, definiendo distinciones de clasificación antropológica de sur a norte en terrenos con mediana inclinación, creciendo los emplazamientos con familias que disfrutan de mejores oportunidades de ingreso, a diferencia de los sectores oriente y occidente caracterizados topográficamente como zonas empinadas y erosionadas, habitadas por familias en situaciones de pobreza, condiciones desventajosas, que padece el proyecto marginal Ciudad Jardín, al estar ubicado en la periferia sur oriental (Hidalgo, 2008). Del mismo modo, Chávez et al. (2018) recomienda olvidar la costumbre tradicional de producir VIS caracterizada por el poco espacio interior y enfocar la oferta de vivienda con soluciones alternativas tendientes a mejorar cualitativamente la VIS, creando espacios para actividades productivas o económicas; por esto, se deben rediseñar los modelos habitacionales con la articulación del sector académico e industrial en los programas de vivienda ofrecidos por el gobierno.

La política de la VIS en Tunja, ha desarrollado programas fragmentados, donde la población ha recurrido al transporte informal para desplazarse hasta las periferias, situación que refleja bajos niveles de movilidad ante la poca frecuencia del servicio de transporte público; adicionalmente, la vivienda se ha entregado con deficientes distribuciones de espacio, que no satisfacen las necesidades elementales de las familias (Cardona et al., 2020). En este contexto, mientras se evada la política de inclusión social, difícilmente se dará el trato merecido a las familias esperanzadas en mejorar su calidad de vida. Aunque el Estado colombiano ha menguado el déficit cuantitativo de VIS con programas tanto de vivienda subsidiada como de VIS gratuita, por ejemplo, 100 mil viviendas gratis y mi casa ya, que a su vez han permitido reactivar la economía con la generación de empleo, aún se requiere que los usuarios se ocupen de la mejora cualitativa de la misma (Otero y Santamaría, 2020). También, el gobierno ha apropiado recursos con destino a la asignación de subsidios a tasas de interés y créditos blandos para aumentar el volumen de la vivienda a familias con un ingreso mensual superior a 2.5 salarios mínimos; esta subvención además de aliviar el costo financiero, aumenta la demanda de vivienda y ofrece la posibilidad al usuario de seleccionar su VIS. 
Con la intención de que los programas VIS se ajusten a las políticas del Estado colombiano, en particular el programa de Ciudad Jardín, Brito et al. (2020) recomiendan a las entidades ejecutoras, coordinar las actividades de servicios al cliente centradas en la adjudicación justa de subsidios, de este modo se puede terminar con los vicios ambiguos demostrados en el incumplimiento de las constructoras a las tipologías reglamentarias y la insostenibilidad de los programas causados por el mismo Estado o por las organizaciones no gubernamentales. Además, la sobrevaloración de la vivienda frente a los demás bienes y la asignación de subsidios a la demanda, benefician más al constructor que a los beneficiarios de la VIS (Camelo y Campo, 2017). Los mecanismos de control deben velar por el acatamiento del reglamento de las políticas de gestión, ejecución y supervisión del manejo de los recursos de la VIS respetando el proceso progresivo de la mejora de la vivienda por adjudicar (Muñoz et al., 2019), sin que esto implique erogaciones adicionales de los usuarios para realizar remodelaciones o ampliaciones para mitigar los espacios reducidos.

La ciudad de Tunja continua su crecimiento urbanístico en el año 1999 con la Ciudadela Ciudad Jardín, proyectando la construcción de 730 unidades VIS; la primera fase para este tipo de vivienda incluyó 306 viviendas subsidiadas y se desarrolló con la práctica habitual de loteo con obras de urbanismo básicas e incompletas bajo el modelo de casas unifamiliares separadas del tejido urbano y con baja calidad constructiva (Hidalgo, 2008). Al respecto, Libertun (2018) afirma que el déficit económico de los hogares los obliga trasladarse a urbanizaciones periféricas construidas por el sector privado con subsidios estatales, proceso que se ha convertido en un común denominador en América Latina y la motivación de los empresarios en urbanizar en la periferia de las ciudades, donde el tiempo para obtener las licencias de construcción es menor, así como el costo y el uso del suelo (Watt y Minton, 2016). La urbanización se interconectó con el resto de la ciudad mediante una vía convencional no pavimentada sin contar con las rutas de transporte público urbano, servicio que parcialmente fue implementado con posterioridad.

Además de la desventaja de que la urbanización Ciudad Jardín esté ubicada en una zona periférica, se adicionan los gastos que representan para el usuario los servicios de educación, salud, recreación y de conexión con los centros comerciales y el resto de la ciudad; condiciones que perdurarán mientras que los programas VIS estén a voluntad de los gobiernos de turno (Salinas y Pardo, 2020). Adicionalmente la urbanización soporta el aislamiento por la presencia de la vía férrea y la doble calzada vial de circulación nacional que dificulta el acceso y pone en riesgo la vida de quienes se movilizan hacia los distintos puntos de la ciudad, también, en el entorno del barrio se presentan altos niveles de inseguridad provocado por el déficit cualitativo de VIS o el hacinamientos provocado por el reducido espacio interior inicial, la falta de servicios básicos suficientes y la escases de áreas recreativas; estas deficiencias son atribuibles también al mercado hipotecario (Monkkonen, 2018).

Wachsmuth y Angelo (2018) consideran que los conflictos entre vecinos son generados en gran parte, por las características físicas de la vivienda, puesto que las construcciones modernas han mantenido criterios tradicionales en su diseño, como una solución a esta situación conflictiva, los propietarios deben participar desde el inicio, en el diseño y la construcción de la vivienda, para que posteriormente no sea necesario hacer modificaciones en sus elementos estructurales y arquitectónicos. Otros aspectos observables están en las dificultades de acceso al barrio, crecimiento urbano irregular, espacio habitable insuficiente y falta de articulación con el espacio público y los equipamientos (Ceballos, 2018). La participación del sector privado en la construcción de la VIS periférica y sus ansias desmedidas por conseguir utilidades grandes, ha reducido el área construida impactando negativamente los parámetros asociados con las características físicoambientales internas (Muñoz et al., 2019), los aspectos estéticos externos y el espacio público en mengua de las condiciones de habitabilidad e iluminación natural de las viviendas (Ceballos, 2018).

\section{METODOLOGÍA}

Este trabajo investigativo se centró en un enfoque empírico-analítico de corte cuantitativo de tipo observacional-descriptivo, de acuerdo con Mendoza et al. (2019), en esta tipología de estudios, los datos se recolectan por medio de variables (características observables) asociadas con un grupo de interés en el propósito de interpretar una realidad social. La información fue recopilada por medio de una encuesta a 78 usuarios, quienes aportaron información sobre las mejoras realizadas a su vivienda. El tamaño de la muestra fue de más del $10 \%$ de la proyección inicial de 730 viviendas, también tuvo en consideración un universo de 306 viviendas construidas en la primera fase, un nivel de confianza del $95 \%$, un error absoluto del $9 \%$ y total incertidumbre en la selección de los encuestados, obteniéndose un tamaño muestral de 85 de los cuales siete usuarios decidieron no responder la encuesta en el último momento. El grupo de estudio fue seleccionado por muestreo aleatorio simple, los participantes dieron su consentimiento informado de manera verbal y de forma voluntaria accedieron a contestar la encuesta constituida por nueve preguntas previamente establecidas, este instrumento fue validado por tres jueces expertos. Con fundamento en el marco teórico, se evaluaron las principales mejoras realizadas por los usuarios de las viviendas ubicadas en el barrio ciudad Jardín, el cual se encuentra ubicado en la periferia de la ciudad de Tunja-Colombia. 
La hipótesis fueron, $\mathrm{H}_{0}$ : las mejoras tanto internas como externas realizadas si están asociadas con los ingresos de los propietarios de las VIS; $\mathrm{H}_{1}$ : las mejoras tanto internas como externas realizadas no están asociadas con los ingresos de los propietarios. El procesamiento de la información se efectuó a través de Excel y el software libre R. Con la información recogida, se hizo un análisis descriptivo acompañado de exégesis textual sobre la observación directa en campo realizada por el investigador principal. Para esto, se generaron tablas de porcentajes provenientes de los datos proporcionados por los encuestados, los cuales permiten la emergencia de los hallazgos, su discusión a la luz del marco teórico y la generación de las conclusiones.

\section{RESULTADOS}

La observación directa del investigador principal permitió establecer que, el barrio denominado Ciudad Jardín está ubicado en la comuna 8, en la calle 10 Sur 2, en la periferia de la ciudad de Tunja en Boyacá-Colombia, en la vía que conduce desde Tunja hacia la población de Soracá en Boyacá, se constituye inicialmente por Viviendas de Interés Social (VIS) de un solo piso cuya fachada es de 3.5 metros de ancho con una puerta metálica y ventana al frente por 3 metros de fondo aproximadamente incluyendo un baño y una pequeña cocina, en lotes de 42 metros cuadrados; el diseño urbanístico no concuerda con los estándares establecidos para la VIS de 135 salarios mínimos, se carece de zonas verdes y las calles al interior del barrio son unidireccionales, lo cual dificulta el tránsito automotor y de las personas; se trata de un barrio con altos niveles de vulnerabilidad.

El $85 \%$ de las viviendas fueron remodeladas por sus propietarios debido a que el espacio interior era insuficiente para albergar a los miembros de la familia; en este contexto, se ha tenido que edificar hacia arriba, varias de las actuales viviendas cuentan hasta con tres pisos, presentan portones metálicos ampliados y ventanales renovados en su perfil frontal; una pocas aún están en ladrillo visto tal como fueron adjudicadas a los beneficiarios. Para mitigar esta situación, la Alcaldía municipal de Tunja ha implementado unos pocos auxilios para efectuar algunas remodelaciones; sin embargo, los usuarios han manifestado verbalmente, haber terminado insatisfechos con las obras y ellos mismos han tenido que hacer erogaciones para mejorar sus viviendas, sacrificando los ingresos destinados a la satisfacción de sus necesidades básicas.

A continuación se presentan los resultados de la encuesta. Con la primera pregunta se pretendía indagar los rangos de ingreso que presentaban los propietarios de la VIS en ciudad Jardín por familia, los usuarios contestaron al interrogante ¿Cuál es el monto de sus ingresos familiares durante el mes?, de la siguiente manera: el $31 \%$ indicó que sus ingresos eran inferiores a un salario mínimo legal vigente en Colombia, el $38 \%$ señaló que era de un salario mínimo, el $21 \%$ de uno hasta dos salarios mínimos, el $9 \%$ de dos a tres salarios mínimos y solamente el $1 \%$ tenía ingresos superiores a tres salarios mínimos. Estos resultados reflejan que el $69 \%$ de las familias tienen ingresos de un salario mínimo o menos. La segunda pregunta se focalizó en determinar la capacidad de ahorro de los usuarios de la VIS, quienes sobre el interrogante ¿Qué porcentaje de sus ingresos mensuales pueden ser destinados al ahorro?, el $36 \%$ indicó que un $5 \%$ de su ingreso mensual, el $39 \%$ señaló que un $10 \%$, el $17 \%$ mencionó que un $15 \%$, el $6 \%$ que un $20 \%$ y solamente el $1 \%$ dijo que más del $20 \%$ del ingreso mensual. Estos hallazgos reflejan una baja capacidad de ahorro, atribuible a que un alto porcentaje de los usuarios tienen ingresos bajos y es casi imposible ahorrar debido a que primero deben sufragar los gastos que implican sus necesidades básicas.

El ítem tres se formuló en referencia a si la vivienda adjudicada por parte del gobierno estaba actualmente habitada. En este contexto, el $66 \%$ mencionó que se encontraba habitada por el propietario, el $31 \%$ señaló que estaba en arrendamiento y el $3 \%$ estaba sin habitar. Estos resultados reflejan que, un $69 \%$ de las VIS estaban habitadas por su propietario o sin habitar; sin embargo un porcentaje considerable de ellas fueron destinadas al arrendamiento. La cuarta pregunta fue ¿A la vivienda adjudicada por el gobierno, usted le ha realizado mejoras? El 78\% indicó que sí le ha hecho mejoras, mientras que el $22 \%$ indicó que no. Estos hallazgos reflejan la capacidad de resiliencia de los propietarios para conseguir el dinero requerido para mejorar su vivienda tanto así que algunos han logrado construir hasta tres pisos, en forma ascendente.

En cuanto a la quinta pregunta ¿Qué tipo de adecuación ha efectuado al interior de su vivienda? De acuerdo con la Tabla 1, respecto a la primera variable, el $29.49 \%$ de los propietarios indicó que ha realizado mejoras en el baño y la cocina, el $7.69 \%$ en la construcción de paredes, el $28.21 \%$ en cambio de pisos, el $25.64 \%$ en instalaciones eléctricas y de agua, el $8.97 \%$ en servicios de internet. Estos resultados indican que la VIS en un alto porcentaje ha requerido de ampliaciones y de mejoras de su infra estructura física. Sobre la sexta pregunta ¿Qué tipo de adecuación ha efectuado en su vivienda, en cuanto a elementos del exterior de su vivienda? En concordancia con la Tabla 1, en referencia a la segunda variable, el $32.05 \%$ indicó que ha efectuado arreglos en la fachada, el $17.95 \%$ en los tejados, el $37.18 \%$ en el gas natural y el $12.82 \%$ en otros aspectos. Se interpreta que también fue necesario que los usuarios efectuaran diversas mejoras externas a su vivienda adjudicada por el gobierno colombiano. 
Tabla 1: Mejoras al interior y exterior de la VIS, propósito y costo de rehabilitar la VIS

\begin{tabular}{|l|l|r|}
\hline Variables & Categorías & Porcentaje \\
\hline \multirow{4}{*}{ Adecuaciones al interior de las VIS } & Mejoras en el baño y la cocina & $29.49 \%$ \\
\cline { 2 - 3 } & Construcción de paredes & $7.69 \%$ \\
\cline { 2 - 3 } & Cambio de pisos & $28.21 \%$ \\
\cline { 2 - 3 } & Instalaciones eléctricas y de agua & $25.64 \%$ \\
\cline { 2 - 3 } & Servicios de internet & $8.97 \%$ \\
\hline \multirow{5}{*}{ Adecuaciones al exterior de las VIS } & Fachada & $32.05 \%$ \\
\cline { 2 - 3 } & Tejado & $17.95 \%$ \\
\cline { 2 - 3 } & Gas natural & $37.18 \%$ \\
\cline { 2 - 3 } & Otros & $12.82 \%$ \\
\hline \multirow{5}{*}{ El para qué se acondicionó la VIS } & Tienda de víveres & $20.52 \%$ \\
\cline { 2 - 3 } & Taller & $8.98 \%$ \\
\cline { 2 - 3 } & Garaje & $38.45 \%$ \\
\cline { 2 - 3 } & Cafetería & $10.26 \%$ \\
\cline { 2 - 3 } & Arrendamiento & $21.79 \%$ \\
\hline \multirow{5}{*}{ Costos para rehabilitar la VIS } & De 1 a 2 millones de pesos colombianos & $14.13 \%$ \\
\cline { 2 - 3 } & De 2 a 5 millones de pesos colombianos & $44.87 \%$ \\
\cline { 2 - 3 } & De 5 a 10 millones de pesos colombianos & $35.90 \%$ \\
\cline { 2 - 3 } & Más de 10 millones de pesos colombianos & \\
\hline
\end{tabular}

En referencia a la séptima pregunta ¿Qué proceso de rehabilitación usted realizó en su vivienda, para qué funciones la acondicionó? En la Tabla 1 se observa que, en cuanto a la tercera variable, el $20.52 \%$ mencionó que acondicionó su VIS para tienda de víveres, el $8.98 \%$ para taller, el $38.46 \%$ para garaje, el $10.26 \%$ para colocar una cafetería y el $21.79 \%$ para arrendamiento. Estos hallazgos evidencian que la VIS se acondicionó para generar ingresos adicionales.El octavo ítem fue ¿Cuál ha sido el costo de rehabilitación de la vivienda en elementos al interior de la misma? Como se observa en la Tabla 1, en lo que respecta a la cuarta variable, el $5.13 \%$ de los propietarios mencionó que el costo de la rehabilitación realizada a su VIS fue de uno a dos millones de pesos porque su capacidad económica más no le permitió, el $14.10 \%$ de dos a cinco millones recurriendo a préstamos, el $44.87 \%$ de cinco a 10 millones mediante ahorro familiar y préstamos, y el $35.90 \%$ indicó que fueron más de 10 millones de pesos provenientes de ahorros, ventas de algunas pertenencias y préstamos. Estos resultados reflejan que, los propietarios han debido realizar grandes esfuerzos económicos para mejorar su vivienda en aspectos internos.

La última pregunta fue ¿Cuál ha sido el costo de rehabilitación de la vivienda en elementos al exterior de la misma? Aproximadamente, el $8.1 \%$ de los propietarios indicó que el costo de rehabilitación de la VIS en aspectos externos fue de uno a dos millones de pesos, el $22.9 \%$ de dos a cinco millones, el $40.3 \%$ de cinco a 10 millones y el $28.7 \%$ indicó que fueron más de 10 millones de pesos. Estos resultados reflejan que, también los propietarios han debido efectuar esfuerzos económicos considerables para mejorar su vivienda en aspectos externos. Estos esfuerzos pudieron ser menores si el gobierno le hubiera adjudicado una VIS en mejores condiciones tanto en los espacios internos como en las características externas.

Para terminar, por medio de una prueba chi-cuadrado se probaron las hipótesis, las cuales mostraron la existencia de una asociación significativa entre las mejoras tanto internas como externas realizadas y los ingresos de los propietarios de las VIS en la urbanización Ciudad Jardín ( $p \_$valor $=0.387$ y $p \_$valor $=0.415$ respectivamente). Los hallazgos y el diálogo directo del investigador principal con los usuarios también proporcionaron evidencias de que los recursos económicos utilizados para hacer las adecuaciones provinieron de diferentes fuentes, entre ellas, el ahorro familiar, los préstamos tanto de entidades financieras como de particulares y en pocos casos algunas ayudas provenientes del gobierno municipal de Tunja contratadas con particulares; estas últimas con bajos niveles de eficacia.

\section{DISCUSIÓN}

Los propietarios reconocen los esfuerzos que ha realizado el gobierno colombiano para proveerles una VIS, la cual fue diseñada y construida con poco espacio habitable y sin tener en cuenta que, no pocas familias eran numerosas y de bajos ingresos económicos. Sin embargo, los propietarios sintieron la necesidad de generar mejoras en sus viviendas de modo que les pudieran acrecentar su nivel de vida (Mendoza et al., 2020) o les permitiera generar algunos ingresos adicionales por medio de pequeños emprendimientos como las tiendas de barrio, las cafeterías, los garajes o los arrendamientos de una parte de su vivienda. De allí la necesidad de haber tomado la decisión de ampliar sus viviendas y construir un piso encima de otro aunque la apariencia arquitectónica no fuera la mejor y diera la idea de crecimiento urbano desordenado (Carreño y Alfonso, 2018). 
En este barrio se observan aspectos que reflejan poca eficiencia gubernamental para gestionar una VIS de calidad que dignifique a las personas que la habitan (Ceballos, 2018) con un diseño arquitectónico que refleja pobreza, construcción urbana periférica y que se constituye en hábitat para personas vulnerables; sus calles están sin pavimentar y las zonas verdes están ausentes; en un alto porcentaje las VIS fueron remodeladas por los propietarios, de tal manera que atendieran sus necesidades personales, aunque en varios casos han debido hacer esfuerzos económicos que han deteriorado la satisfacción de sus necesidades básicas (Chávez, Pérez y Serrano, 2018), los cuales han implicado recurrir a un limitado ahorro familiar, los préstamos bancarios o a los subsidios del gobierno municipal destinados a la mejora cualitativa de la vivienda.

En este contexto, las soluciones de vivienda adjudicadas por el gobierno en la urbanización ciudad Jardín en Tunja fueron diseñadas con un espacio insuficiente para ser habitadas por núcleos familiares constituidos por más de dos personas; este tipo de hábitat genera un sinnúmero de problemas para sus beneficiarios tanto en la salud como en aspectos económicos y sociales (Mendoza et al., 2021); asimismo, esta clase de urbanización periférica produce diversos problemas relacionados con el desarrollo de la ciudad tales como: la fragmentación urbana, la dificultad de interconexión con otros puntos del sector urbano, la calidad ambiental del barrio, los impactos asociados con la adecuación de las viviendas a las necesidades domésticas, de sociabilidad, realización de actividades tan laborales como productivas y posibilidad de acceder a centros educativos, comerciales y de salud ubicados dentro de la ciudad, además de la baja posibilidad de acceder a los microcréditos para mejorar su vivienda generada por la falta de respaldo ante las entidades financieras (Lehner y Gerscovich, 2021).

Los actores urbanos ubicados en esta periferia de la ciudad de Tunja manifiestan su voz de inconformidad con los gobiernos de turno que, poco han hecho por mejorar sus condiciones de habitabilidad, teniendo que recurrir a su propio esfuerzo económico para mejorar su vivienda de manera cualitativa (Hidalgo, 2008) tanto en aspectos internos como externos de su VIS en la búsqueda de un mejor recinto para vivir, donde la necesidad de ingresos adicionales prima sobre la estética de la urbanización (Muñoz et al., 2019) y el crecimiento acelerado de la ciudad. Este trabajo aporta información adicional en el sentido que, proporciona evidencias de las desigualdades e inequidades que viven los beneficiarios en los diversos proyectos de VIS (Mendoza et al., 2020) implementados en la ciudad de Tunja o en otros desarrollados en diferentes ciudades del país, que ameritarían ser analizadas a través de estudios comparativos tanto en sus diseños arquitectónicos como en la calidad de las VIS entregadas a los beneficiarios por el gobierno.

\section{CONCLUSIONES}

Con base en la observación directa y los resultados reflejados por la encuesta, se concluye que: 1) en la urbanización ciudad Jardín existen calles sin pavimento, estructuras arquitectónicas heterogéneas con apariencias diversas, cuyos diseños originales con poco espacio construido para ser habitable, han debido modificarse con construcciones hacia arriba, similares a edificios urbanos pequeños que contrastan con la apariencia de una ciudad planificada y generan vulnerabilidad a sus habitantes, 2) las modificaciones realizadas por los usuarios obedecen a la necesidad de generar ingresos adicionales para sus propietarios, quienes en un alto porcentajes son personas de estratos socioeconómicos bajos caracterizados por ingresos inferiores a dos salarios mínimos, con poca capacidad de ahorro por cuanto su ingreso ha de direccionarse a sufragar los gastos asociados con la satisfacción de sus necesidades primarias, 3) el gobierno ha tenido la voluntad de apoyar la vivienda VIS en condiciones de habitabilidad, sin embargo la empresa contratista y los organismos de control, han mostrado bajos niveles de eficiencia, haciendo evidente el descontento de las familias beneficiarias, 4) en un alto porcentaje, con el esfuerzo económico realizado por los usuarios, se ha mejorado la habitabilidad y funcionabilidad de las VIS adjudicadas por el gobierno, 5) los propietarios instan al gobierno para que evite la implementación de programas VIS fragmentados, con diseños en espacios reducidos ubicados en la periferia porque acentúan la desigualdad social en los beneficiarios e implican esfuerzos económicos familiares para rediseñar sus viviendas; además reclaman mejoras en la infraestructura del barrio y en la interconexión con el resto de la ciudad.

\section{REFERENCIAS}

Adewale, B.A., Ibem, E.O., Amole, S. A., y Adeboye, A.B., Place attachment in Nigerian urban slums: Evidence from inner-city Ibadan, https://doi.org/10.1016/j.cities.2020.102902, Cities, 107, 102902 (2020)

Brito, C., Pitre, R., y Cardona, D., Clima organizacional y su influencia en el Desempeño del personal en una Empresa de Servicio, http://dx.doi.org/10.4067/S0718-07642020000100141, Información Tecnológica, 31(1), 141-148 (2020)

Camelo, M., y Campo, J., Modelo de consumo de Vivienda óptimo: Estimaciones para Bogotá, https://doi.org/10.18359/rfce.2655, Revista de la Facultad de Ciencias Económica: Investigación y Reflexión, 25(1), 105-116 (2017) 
Cardona, S., Escobar, D., y Moncada, C., Análisis de Ordenamiento de la Movilidad en el Bulevar gastronómico de Milán, Manizales (Colombia), http://dx.doi.org/10.4067/S0718-07642020000100301, Información Tecnológica, 31(1) 301 $310(2020)$

Carreño, C., y Alfonso, W., Relación entre los Procesos de urbanización, el Comercio internacional y su incidencia en la Sostenibilidad urbana, http://dx.doi.org/10.11144/javeriana.cvu11-22.rpuc, Cuadernos de Vivienda y Urbanismo, 11(22), $1-29(2018)$

Ceballos, O., La Infancia y el Hábitat urbano informal. Reflexiones sobre Bogotá-Colombia, https://doi.org/10.15446/bitacora.v28n1.57890, Bitácora Urbano Territorial, 28(1), 85-92 (2018)

Chávez, M., Pérez, D.D., y Serrano, M.F., Impact on the direct Costs of Social Interest and Priority interest Housing by including new Construction Standards: Cali case, http://doi.org/10.15446/dyna.v85n206.69013, Dyna, 85 (206), $31-38$ (2018)

Chiodelli, F., y Moroni, S., Planning, Pluralism and religious Diversity: Critically reconsidering the Spatial regulation of Mosques in Italy starting from a much debated law in the Lombardy region, https://doi.org/10.1016/j.cities.2016.12.004, Cities, 62, 62-70 (2017)

Davies, J. S., y Blanco, I., Austerity urbanism: Patterns of Neoliberalisation and Resistance in six Cities of Spain and the UK, https://doi.org/10.1177/0308518X17701729, Environment and Planning A, 49(7), 1517-1536 (2017)

García, F., Smith, H., y Calderon, E., Contemporary Tendencies in Colombian urban Planning: the case of the 'Planes Parciales' in Medellín, https://doi.org/10.1080/13563475.2018.1500276, International Planning Studies, 23(4), 355- 375 (2018)

Hidalgo, A., El Papel de la Vivienda en la Configuración Urbana de las Periferias: caso de Tunja-Colombia 1907-2007,

Cuadernos de vivienda y urbanismo, ISSN-e 2145-0226, 1(1), 12-43 (2008)

Lehmann, P., Brenck, M., Gebhardt, O., Schaller, S., y Süßbaue, E., Barriers and Opportunities for Urban adaptation planning: analytical framework and evidence from Cities in Latin America and Germany, https://doi:10.1007/s11027-0139480-0, Mitig Adapt Strateg Glob Change, 20, 75-97 (2015)

Lehner, J. M., y Gerscovich, A., Contrasting housing Microfinance with the Social production of Habitat in the City of Buenos Aires, Argentina, https://doi.org/10.1080/19491247.2021.1906484, International Journal of Housing Policy, 21, 1$16(2021)$

Libertun, N., The social Housing burden: Comparing households at the Periphery and the Center of cities in Brazil, Colombia, and Mexico, https://doi.org/10.1080/19491247.2017.1298366, International Journal of Housing Policy, 18(2), 177-203 (2018)

Mendoza, H.H., Burbano, V.M., y Valdivieso, M.A., El Rol del Docente de Matemáticas en Educación Virtual Universitaria. Un Estudio en la Universidad Pedagógica y Tecnológica de Colombia, http://dx.doi.org/10.4067/S071850062019000500051, Formación universitaria, 12(5), 51-60 (2019)

Mendoza, J.M., Burbano, V.M., y Mendoza, H.H., Enseñanzas atribuibles a los Procesos de Adjudicación de Vivienda de Interés Social en una ciudad colombiana: grado de satisfacción del usuario, http://dx.doi.org/10.4067/S071807642020000500119, Información tecnológica, 31(5), 119-118 (2020)

Mendoza, J.M., Burbano, V.M., y Mendoza, H.H., Evaluación de la Vivienda de Interés Prioritario desde la Perspectiva de los Beneficiarios: urbanización Antonia Santos, Tuna, Colombia, http://dx.doi.org/10.4067/S071807642021000400157, Información tecnológica, 32(4), 157-166 (2021)

Monkkonen, P., Do we need innovation in housing policy? Mass production, Community-based upgrading, and the Politics of urban land in the Global South, https://doi:10.1080/19491247.2017.1417767, International Journal of Housing Policy, 18(2), 167-176 (2018)

Muñoz, L., Arcila J., y otros 4 autores, Una estética de la Vivienda de Interés Social: Desarrollos progresivos en PalmiraColombia (2000-2017), https://doi:10.4067/S071883582020000100075, Revista INVI, 35(98), 75-100 (2019)

Murray, C., y Clapham, D., Housing policies in Latin America: Overview of the four largest Economies, https://doi.org/10.1080/14616718.2015.1055090, International Journal of Housing Policy, 15(3), $347-364$ (2015)

Otero, K. L. M., y Santamaría, J. E. V., La Apropiación Social y Espacial de Vivienda de Interés Prioritario (VIP): Casos Las Flores y La Aurora en la Ciudad de Medellín, https://doi.org/10.46743/2160-3715/2020.4690, The Qualitative Report, 25(7), 1960-1980 (2020)

Rolnik, R., Late neoliberalism: The financialization of Homeownership and Housing rights, https://doi.org/10.1111/14682427.12062, International Journal of Urban and Regional Research, 37(3), 1058-1066. (2013)

Ruá, M. J., Huedo, P., Civera, V., y Agost, R., A simplified Model to assess vulnerable Areas for Urban regeneration, https://doi.org/10.1016/j.scs.2019.101440, Sustainable Cities and Society, 46, 101440 (2919)

Salinas, L., y Pardo, A., Política de vivienda y Habitabilidad en la periferia de la Zona Metropolitana del Valle de México, http://dx.doi.org/10.4067/S0718-34022020000200051, Revista De Geografía Norte Grande, (76), 51-69 (2020) 
Soares, F., Santos, J., y otros 3 autores, Tipología socio-ambiental de las Ciudades medias de Brasil: aportes para un Desarrollo urbano sostenible, https://doi.org/10.1590/2175-3369.008.002.AO08, Urbe, Revista Brasileira de Gestão Urbana, 8(2), 272-287 (2016)

Timo von Wirth, Fuenfschilling, L., Frantzeskaki, N., y Coenen, L., Impacts of urban living labs on Sustainability transitions: Mechanisms and Strategies for systemic change through Experimentation,

https://doi.org/10.1080/09654313.2018.1504895, European Planning Studies, 27(2), 229-257 (2019)

Valdivia, A. L., Obstáculos Epistemológicos en Urbanismo, http://dx.doi.org/10.11144/Javeriana.cvu12-23.oeeu, Cuadernos de Vivienda y Urbanismo, 12(23), 1-19 (2018)

Vázquez, J., Montalvo, R., Amézquita, J., y Arredondo, F., El ahorro en la carencia. Una reflexión sobre los Hábitos de Ahorro de familias de una Zona vulnerable de México, Revista Perspectivas, ISSN 1994-3733, 19 (39), 103-120 (2017)

Wachsmuth, D., y Angelo, H., Green and gray: New ideologies of Nature in Urban sustainability Policy, https://doi.org/10.1080/24694452.2017.1417819, Annals of the American Association of Geographers, 108(4), 10381056 (2018)

Watt, P., y Minton, A., London's Housing crisis and its Activisms, https://doi.org/10.1080/13604813.2016.1151707, City, 20(2), 204-221 (2016) 